\title{
Complications chirurgicales de la stimulation cérébrale profonde : expérience clinique à propos de 184 cas
}

(Surgical complications of deep brain stimulation: Clinical experience of 184 cases)

\author{
Mendes-Martins V. ${ }^{\mathrm{a},{ }^{*}}$, Coste J. ${ }^{\mathrm{a}, \mathrm{d}}$, Derost P. ${ }^{\mathrm{b}}$, Ulla M. ${ }^{\mathrm{b}}$, Gabrillargues J., ${ }^{\mathrm{c}, \mathrm{d}}$, \\ Durif F. ${ }^{\mathrm{b}}$, Chazal J., ${ }^{\mathrm{a}, \mathrm{d}}$, Lemaire JJ.,
}

a - Service de neurochirurgie A, hôpital Gabriel-Montpied, CHU de Clermont-Ferrand, 28, rue Montalembert, 63003 Clermont-Ferrand cedex 1, France

b - Service de neurologie, CHU de Clermont-Ferrand, 28, rue Montalembert, 63003 Clermont-Ferrand, France

c - Unité de neuroradiologie, CHU de Clermont-Ferrand, 28, rue Montalembert, 63003 Clermont-Ferrand, France

d - EA 3295, équipe de recherche en signal et imagerie médicale, Image-Guided Clinical Neuroscience and Connectomics (IGCNC), Clermont université, université d'Auvergne, BP 10448, 63000 Clermont-Ferrand, France

* - Corresponding author : vmendesmartins@ch-annecy.fr

\begin{abstract}
Background and purpose Deep brain stimulation (DBS) is an effective treatment of movement disorders and psychiatric diseases. However, this surgery is still time consuming and associated with complications, among which we aimed to identify non-stimulo-induced adverse effects. Hence, we retrospectively systematically analyzed patients operated on, at our institution, using magnetic resonance imaging (MRI) direct anatomic mapping.

Methods One hundred and seventy-five patients (184 surgeries) were performed between 1994 and 2008, for Parkinson's disease, essential tremor, dystonia and obsessive compulsive disorder. Primary anatomic targets were the subthalamic region, the intern pallidum and the thalamus. Final electrode positioning was adjusted according to intraoperative neuron recordings and acute stimulation tests. All surgically related adverse effects were collected. Life threatening or new non-planed surgery was considered as severe adverse effects.
\end{abstract}

Results Adverse effects occurred 10 times (5.4\%) during MRI acquisition, 24 times (13\%) with five serious $(2.7 \%)$ including one1 death $(0.5 \%)$ during electrodes implantation and 17 times $(9.2 \%)$ with four serious $(2.2 \%)$ during neuropacemaker implantation. Electrodes were repositioned in six cases (five patients, $\mathrm{n}=3.4 \%$ ).

Conclusion DBS efficiency is recognized however the significant incidence of adverse effects should prompt us to improve the procedures. 


\section{Résumé}

Introduction La stimulation cérébrale profonde est un traitement reconnu des mouvements anormaux et de certains troubles psychiatriques. Néanmoins, il s'agit toujours d'une procédure chirurgicale longue, délicate et à l'origine de complications. Nous avons analysé les évènements indésirables non stimuloinduits survenus sur 14 ans au CHU de Clermont-Ferrand où nous réalisons une visée stéréotaxique directe à partir d'une imagerie par résonance magnétique nucléaire (IRM).

Patients et méthode Cent soixante-quinze patients (184 interventions) ont été réalisés entre 1994 et 2008 pour : maladie de Parkinson idiopathique, tremblement essentiel, dystonie et trouble obsessionnel compulsif. Les cibles anatomiques primaires visées étaient : la région sous-thalamique, le pallidum interne ou le thalamus. Les électrodes ont été positionnées après enregistrement neuronal et tests aigus de stimulation. Tous les évènements indésirables ont été recueillis et analysés rétrospectivement ; l'engagement du pronostic vital ou une ré-intervention non programmée ont été considérés comme évènements indésirables graves.

Résultats Le taux d'évènements indésirables par intervention était de 5,4 \% lors de la réalisation de l'IRM (dix cas), de $13 \%$ lors du positionnement d'électrodes (24 cas ; cinq cas graves incluant 1 décès, 0,5\%), et de $9,2 \%$ lors de l'implantation du neurostimulateur (17 cas ; quatre graves, 2,2\%). Cinq patients (six chirurgies ; $3,4 \%$ ) ont eu un repositionnement d'électrodes.

Conclusion L'efficacité de la stimulation cérébrale profonde n'est plus à prouver, mais la fréquence non négligeable des évènements indésirables doit nous inciter à améliorer les procédures.

Keywords: Deep brain stimulation, Surgical complications, Direct targeting, Thalamus, Subthalamic nucleus, Globus pallidus internus

Mots clés : Stimulation cérébrale profonde, Complications chirurgicales, Visée directe, Thalamus, Noyau sous-thalamique, Globus pallidum interne 


\section{Introduction}

La stimulation cérébrale profonde ( $\mathrm{SCP}$ ) est une alternative thérapeutique au traitement médical de mouvements anormaux, et en cours d'évaluation pour certains troubles psychiatriques (Blond et al., 2000). Elle s'appuie sur de très nombreux travaux concernant les bénéfices cliniques et les effets indésirables stimulo-induits (Deuschl et al., 2006 ; Hariz et al., 2008 ; Krack et al., 2003 ; Rodriguez-Oroz et al., 2005). Les complications liées à la procédure chirurgicale ont été peu rapportées (Hariz et al., 2008 ; Joint et al., 2002 ; Lyons et al., 2004 ; Oh et al., 2002 ; Seijo et al., 2007 ; Umemura et al., 2003). La chirurgie de SCP est codifiée et maîtrisée par les équipes qui la pratiquent couramment mais reste une procédure relativement longue et délicate. Dans un souci d'évaluation de nos pratiques et pour compléter les données de la littérature, nous avons étudié le taux d'évènements indésirables (EI) survenus au cours de 184 interventions sur une période de 14 ans, au CHU de Clermont-Ferrand. Nous pratiquons systématiquement un ciblage direct primaire sur une imagerie par résonance magnétique (IRM), ceci pour une analyse fine de la fonction, de l'électrophysiologie et des structures anatomiques, et une réduction du nombre de trajectoires d'exploration.

\section{Population et méthode}

\subsection{Population}

Entre 1994 et 2008, 175 patients (113 hommes, 65 \%), ont été opérés pour une SCP ; l'âge moyen était de 60 ans $\pm 10,4$ années (de neuf à 83 ans), et l'évolution moyenne de la maladie était de 11 ans $\pm 7,0$ années (de un à 57 ans). Trois cent cinquante et une électrodes ont été implantées au cours de 184 procédures (Tableau 1). La cible anatomique primaire était la région du noyau sous-thalamique (NST, 140 cas), le thalamus moteur ventral (noyau ventral intermédiaire médian, Vim, 23 cas ; noyau ventral antérieur Va, un cas), et le pallidum interne (Globus Pallidus internus, GPi, 19 cas). Les indications pour une primoimplantation étaient les complications motrices de la maladie de Parkinson idiopathique sévère (154 cas ; deux interventions sous anesthésie locale n'ont pu être menées à leur terme, en raison d'un état anxieux sévère du patient), de la dystonie (primaire, trois cas, et secondaire, sept cas) et du tremblement essentiel (huit cas) ou secondaire (un post anoxique et un lors de sclérose en plaque); un trouble obsessionnel compulsif (NST) a été traité dans le cadre d'un protocole de recherche. Cinq patients (dont un à deux reprises) ont été réopérés pour un contrôle insuffisant des symptômes moteurs, en moyenne 36,8 mois \pm 23,7 mois, après la première intervention, un autre pour une dysarthrie importante, un pour contrôler une dystonie unilatérale (GPi) apparue à distance de la première chirurgie (NST), et un patient pour traiter le côté controlatéral (Tableau 2). 


\begin{tabular}{|c|c|c|c|c|}
\hline Indication & \multicolumn{4}{|c|}{ Nombre d'électrodes Nombre de patients Sous-total Total des patients } \\
\hline \multicolumn{5}{|l|}{ Première intervention } \\
\hline \multicolumn{5}{|l|}{ MPI } \\
\hline Chirurgie interrompue & n.a. & 2 & 2 & 2 \\
\hline NST & 267 & 134 & 152 & 173 \\
\hline GPi & 15 & 8 & & \\
\hline Vim & 16 & 10 & & \\
\hline \multicolumn{5}{|l|}{ TE } \\
\hline Vim & 13 & 8 & 10 & \\
\hline \multicolumn{5}{|c|}{ Tremblements secondaires } \\
\hline Vim & 4 & 2 & & \\
\hline \multicolumn{5}{|l|}{ Dystonie $\mathrm{I}^{\text {aire }}$} \\
\hline GPi & 3 & 2 & 10 & \\
\hline $\mathrm{GPi}+\mathrm{Vim}$ & 2 & 1 & & \\
\hline \multicolumn{5}{|l|}{ Dystonie II ${ }^{\text {aire }}$} \\
\hline GPi & 12 & 6 & & \\
\hline $\mathrm{Va}$ & 2 & 1 & & \\
\hline \multicolumn{5}{|l|}{ TOC } \\
\hline NST & 2 & 1 & 1 & \\
\hline \multicolumn{5}{|l|}{ Ré-intervention } \\
\hline \multicolumn{5}{|l|}{ MPI } \\
\hline NST & 9 & 5 & 6 & 9 \\
\hline GPi & 2 & 1 & & \\
\hline \multicolumn{5}{|l|}{ TE } \\
\hline Vim & 3 & 2 & 2 & \\
\hline \multicolumn{5}{|l|}{ Dystonie II $^{\text {aire }}$} \\
\hline GPi & 1 & 1 & 1 & \\
\hline Total & 351 & & 184 & \\
\hline
\end{tabular}

Tableau 1. Interventions de stimulation cérébrale profonde.

MPI : maladie de Parkinson idiopathique ; TE : tremblement essentiel ; TOC : trouble obsessionnel compulsif ; NST : noyau sous-thalamique ; GPi : globus pallidus interne ; Vim : noyau ventral intermédiaire; Va: noyau ventral oral. 


\begin{tabular}{|c|c|c|c|c|c|c|c|}
\hline \multicolumn{4}{|c|}{ Intervention initiale } & \multicolumn{4}{|c|}{ Ré-intervention } \\
\hline Indication & & Cible & Imagerie & Indication & Intervention & Cible & e IRM \\
\hline MPI & unilatéral & 1 Vim & Ventriculographie & \multirow{6}{*}{$\begin{array}{l}\text { Signes moteurs } \\
\text { mal contrôlés }\end{array}$} & Bilatéral & NST & DP \\
\hline MPI & bilatéral & GPi & IRM (DP) & & Bilatéral & NST & DP \\
\hline MPI & bilatéral & NST & Ventriculographie & & Bilatéral * & NST & DP \\
\hline MPI & bilatéral & NST & IRM (DP) & & Unilatéral & NST & DP \\
\hline TE & bilatéral & Vim & IRM (TSE) & & Bilatéral & Vim & TSE \\
\hline MPI & bilatéral & NST & IRM (WAIR) & & Bilatéral & NST & WAIR \\
\hline MPI & bilatéral & NST & IRM (DP) & Dysarthrie stimulo-induite & Bilatéral & NST & TSE \\
\hline MPI & bilatéral & NST & IRM (WAIR) & Dystonie non stimulo-induite & Unilatéral & GPi & WAIR \\
\hline TE & unilatéral & Vim & Ventriculographie & 2e côté & Unilatéral & Vim & TSE \\
\hline
\end{tabular}

Tableau 2. Patients réopérés $(n=8)$ d'une stimulation cérébrale profonde.

MPI : maladie de Parkinson idiopathique ; TE : tremblement essentiel ; TOC : trouble obsessionnel compulsif ; NST : noyau sous-thalamique ; GPi : globus pallidus interne ; Vim : noyau ventral intermédiaire ; DP : séquence en densité de protons ; TSE : séquence turbo spin echo ; WAIR : séquence d'inversion récupération atténuant la substance blanche. * Patient réopéré deux fois.

\subsection{Technique chirurgicale}

Le ciblage probabiliste indirect sur ventriculographie a été réalisé 12 fois au début de notre expérience. Il a été progressivement remplacé par un ciblage anatomique primaire sur IRM en conditions stéréotaxiques permettant d'identifier directement des structures anatomiques (172 ciblages primaires directs); il a été réalisé pour la première fois pour viser le pallidum interne (Lemaire et al., 1999).

L'IRM stéréotaxique était réalisée avec un cadre Leksell (Leksell G frame, Elekta, Suède) et son kit de repositionnement (Leksell repositioning kit, Elekta, Suède), le patient étant sevré de son traitement antiparkinsonien. L'IRM a été conduite sous anesthésie locale dans $98,4 \%$ des cas. La première séquence IRM utilisée était en densité de proton avec un imageur à 1 Tesla (48 patients ; Magnetom® Siemens, Erlangen, Allemagne), secondairement adaptée à un imageur 1.5 Tesla (Sonata ${ }^{\circledR}$ Siemens, Erlangen, Allemagne), puis remplacée par une séquence turbo spin echo (TSE) pondérée en T2 (42 fois). Enfin, nous avons développé une séquence en inversion récupération à 1.5 Tesla, spécifique à l'exploration des ganglions de la base et du thalamus contrastant spontanément la substance blanche et la substance grise, sans distorsion significative, (Sonata ${ }^{\circledR}$ et Avanto ${ }^{\circledR}$, Siemens, Erlangen, Allemagne) ; nommée White Matter Attenuated Inversion Recovery (WAIR, 69 acquisitions) (Lemaire et al., 2007). Après l'acquisition des images, le cadre de stéréotaxie a été retiré pour le confort du patient car l'implantation des électrodes avait lieu le lendemain. Le planning chirurgical a été effectué entre temps avec un logiciel de chirurgie stéréotaxique (@target@ 1.18, puis Iplan® 1.1-2.6 ; BrainLAB, Feldkirchen, Allemagne) (Lemaire et al., 2004). 
Le lendemain, après repositionnement du cadre de stéréotaxie, l'implantation des électrodes de SCP a été réalisée en un seul temps. La trajectoire planifiée optimisée a été réalisée selon un protocole standardisé :

- $\quad$ orifice osseux de $6 \mathrm{~mm}$ en regard de la seconde circonvolution frontale ;

- $\quad$ micro-électrodes (FHC World Precision Instruments Inc., Sarasota, États-Unis ; Alpha Omega Engineering, Nazareth, Israël) introduites dans un tube guide après perforation durale sans fuite de liquide cérébrospinal (Bengun, Immi ; France), jusqu'à $10 \mathrm{~mm}$ de la cible primaire, sur plusieurs trajectoires (en moyenne 2,4 par côté ; 2,2 pour la visée du GPi, 2,3 pour la visée du Vim, et 2,5 pour la visée du NST), parallèles et distantes de $2 \mathrm{~mm}$ autour de la trajectoire centrale planifiée ;

- $\quad$ exploration électrophysiologique motorisée enregistrant d'abord l'activité spontanée neuronale tous les $0,5 \mathrm{~mm}$, pendant 30 secondes (pointe d'enregistrement en tungstène, diamètre $=25 \mu \mathrm{m}$, longueur $=100 \mu \mathrm{m}$ ), puis une seconde exploration de la même zone à la recherche des effets cliniques (bénéfices et effets indésirables) en stimulation (diamètre $550 \mu \mathrm{m}$, longueur 1,2 $\mathrm{mm}$ et surface 2,1 $\mathrm{mm} 2$, avec une densité de courant maximale de $1,9 \mathrm{~mA} / \mathrm{mm} 2$, fréquence de $130 \mathrm{~Hz}$ et durée d'impulsion de $60 \mu \mathrm{s}$ ), tous les millimètres par pallier de $0,2 \mathrm{~mA}$, dans la limite de $3 \mathrm{~mA}$.

Le site optimal de stimulation correspondait à l'endroit où était obtenu le meilleur effet clinique, avec la plus faible intensité de stimulation, et offrant la grande amplitude d'intensité avant l'apparition d'effets indésirables.

L'électrode de SCP définitive (quadripolaire électrodes DBS 3387, puis 3389, Medtronic Inc, États-Unis) a été placée de telle sorte que le contact 1 soit sur le site optimal de stimulation, puis fixée, sous contrôle radiographique, avec une mini plaque d'ostéosynthèse (Minitek, Tekka SA, France) et un bouchon d'étanchéité en Silastic maintenu à l'Histoacryl ${ }^{\circledR}$. Vingt-cinq interventions se sont déroulées en position assise au début de notre expérience, puis 158 en position allongée, $123(68,7 \%)$ sous anesthésie locale, 51 $(28,5 \%)$ sous neuroleptanalgésie et $5(2,8 \%)$ sous anesthésie générale. Le temps moyen d'implantation pour une procédure standard bilatérale a été de sept heures, diminuant progressivement avec l'expérience.

La position des contacts de l'électrode définitive par rapport au ciblage anatomique préopératoire a été systématiquement vérifiée par des radiographies stéréotaxiques per opératoire (153 cas), par IRM postopératoire stéréotaxique (108 cas), puis par tomodensitométrie non stéréotaxique (29 cas). Un générateur externe a été utilisé temporairement pour 13 patients pour tester l'efficacité des électrodes ; cette technique a été utilisée au début de la chirurgie de SCP.

Quelques jours (deux à cinq jours en moyenne) après l'implantation des électrodes, en l'absence de syndrome infectieux, un neurostimulateur (Itrel II 7424, Soletra 7426, puis Kinetra 7428, Medtronic Inc, États-Unis) a été mis en place sous anesthésie générale, dans la région sous-claviculaire (181 patients) ou abdominale (50 patients).

Tous les patients ont bénéficié d'un suivi sur le long terme en collaboration avec l'équipe de neurologie et de neuroradiologie du CHU de Clermont-Ferrand. 


\subsection{Analyse des événements indésirables non stimulo-induits liés à la chirurgie}

Les événements indésirables non stimulo-induits ont été répartis en trois catégories : ceux survenus lors de l'implantation des électrodes, ceux survenus lors de la mise en place du neurostimulateur et ceux directement dus à un problème technique (imageur, cadre de stéréotaxie, micromoteur). Nous avons distingué les EI graves définis comme mettant en jeu le pronostic vital et/ou nécessitant d'une réintervention non programmée (Moore et al., 2007).

L'influence de l'âge ( $\leq 60$ ans, $>60$ ans), de la pathologie traitée (MPI, TE ou dystonie), de la cible anatomique (NST, Vim, GPi), du nombre de trajectoires $(\leq 2,>2)$, de la position opératoire (assise, décubitus dorsal), de la durée d'intervention, et du type d'imagerie préopératoire (téléventriculographie stéréotaxique, IRM en conditions stéréotaxiques, séquences TSE et WAIR) a été analysée avec un test de Chi2 de Pearson ou un test exact de Fisher si l'effectif était inférieur à 5, à l'aide du logiciel R2.12.1 (www.r-project.org). Le risque de première espèce $\alpha$ était fixé à $5 \%$.

\section{Résultats}

\subsection{Morbi-mortalité de la chirurgie d'implantation des électrodes}

Les complications, rapportées au nombre d'interventions, ont été étudiées en fonction du temps (Fig. 1). Globalement, 24 EI $(13 \%)$, dont quatre graves (2,2 \%), sont survenus lors de l'implantation des électrodes.

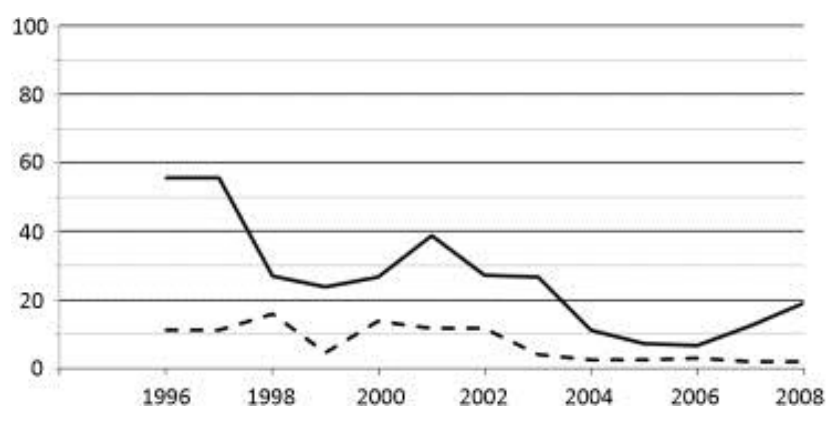

Figure 1. Pourcentage de complications (moyenne glissante sur trois ans), survenues au cours des 184 procédures chirurgicales de stimulation cérébrale profonde, lors de la mise en place des électrodes (trait plein) et la mise en place du neurostimulateur (pointillés). NB : le taux de complications est supérieur à $50 \%$ sur la moyenne des trois premières années, car il est calculé pour six patients opérés en position assise.

Les quatre EI graves $(2,2 \%)$ étaient : un décès 12 heures après l'intervention $(0,5 \%)$ sans cause identifiée (tomodensitométrie post-mortem normale ; refus de l'autopsie), deux infections avec retrait de la totalité du matériel $(1,1 \%)$, et un hématome intracérébral $(0,5 \%)$ nécessitant une évacuation chirurgicale en 
urgence. Il était survenu après enregistrement électrophysiologique sur cinq micro-électrodes lors d'un protocole de recherche.

Dix-neuf EI non graves ont été notés : six épisodes de confusion per opératoire (3,3\%), quatre épisodes de dyspnées $(2,2 \%)$, trois infections (un désunion de cicatrice, deux infections urinaires) traitées médicalement $(1,6 \%)$, un crise d'épilepsie tonico-clonique généralisée $(0,5 \%)$ observée pendant l'enregistrement électrophysiologique, mais sans anomalie tomodensitométrique, un suffusion hémorragique sous-durale asymptomatique $(0,5 \%)$, visualisée sur l'imagerie postopératoire systématique, et quatre positionnements non optimal de l'électrode (2,2\%). Il s'agissait d'une mauvaise direction prise par l'électrode $(\mathrm{n}=3)$, et d'une fracture de la collerette d'histo-acryl avant fixation de l'électrode $(\mathrm{n}=1)$.

Enfin, une procédure a dû être interrompue en raison d'une anxiété empêchant toute collaboration du patient pendant la phase d'exploration électrophysiologique $(0,5 \%)$.

Il y a eu significativement plus de complications en position assise que couchée $(p=0,02)$, notamment la dyspnée peropératoire $(\mathrm{p}=0,003)$. Les risques hémorragiques ne sont pas influencés par l'âge $(\mathrm{p}=0,062$, ni par la cible visée $(p=0,73)$ ni par le nombre de trajectoires d'exploration électrophysiologique $(\mathrm{p}=$ $0,82)$. Le risque infectieux n'est pas augmenté par l'âge $(\mathrm{p}=0,56)$, ni par la cible visée $(0,69)$.

Six ré-interventions chez cinq patients ont été nécessaires pour un contrôle insuffisant des symptômes moteurs $(\mathrm{n}=3,4 \%)$, sans lien significatif avec l'imagerie préopératoire $(\mathrm{p}=0,2)$, ni avec la cible anatomique $(\mathrm{p}=0,07)$ (Tableau 2). À trois reprises, la cible anatomique a pu être plus précise avec une imagerie plus appropriée. Dans un cas, nous sommes passés d'une visée indirecte du Vim à une visée directe. Dans un autre cas, nous avons traité une seconde cible (GPi puis NST). Dans le dernier cas, il s'agissait d'un ciblage volontairement plus postérieur au sein du NST, en accord avec les données de la littérature (Plaha et al., 2006), mais qui n'a pas été efficace.

\subsection{Morbidité de la chirurgie d'implantation du neurostimulateur}

Dix sept EI (9,2\%) ont été notés lors de la chirurgie d'implantation du neurostimulateur; dont six graves $(3,3 \%)$ par réintervention non programmée (Moore et al., 2007) : un hématome $(0,5 \%)$, trois infections $(1,6 \%)$ et deux fractures du câble prolongateur $(1,1 \%)$. Trois autres hématomes de la poche d'implantation $(1,6 \%)$ ont été traités médicalement, et huit patients $(4,3 \%)$ ont décrit une gêne liée au volume de l'implant ou à la tension entraînée par le câble prolongateur, que le neurostimulateur soit en position abdominale ou sous-claviculaire.

\subsection{Morbidités en lien avec les appareils utilisés lors de la procédure chirurgicale}

L'acquisition de l'imagerie pré opératoire s'est effectuée sans EI grave. Un examen $(0,5 \%)$ n'a pu être réalisé et trois autres $(1,6 \%)$ ont nécessité une neuroleptanalgésie ou une anesthésie générale, en raison de mouvements anormaux trop intenses. Des troubles respiratoires (deux patients, 1,1\%), des troubles métaboliques (un patient, 0,5\%), des troubles de la statique rachidienne (quatre patients, 2,2 \%) ont prolongé l'acquisition IRM. 
D'autres problèmes techniques ont entravé sept procédures $(3,8 \%)$ : liés à l'IRM panne, (un patient, 0,5 $\%)$; impossibilité de faire une imagerie sagittale par encombrement de la tête dans le cadre, (un patient, $0,5 \%)$; liés au cadre de stéréotaxie par défaut d'ancrage du système de repositionnement du cadre (un patient, $0,5 \%$ ) ; usure de pièce du cadre entraînant une imprécision de visée, (deux patients, 1,1 \%) ; et liés au matériel d'exploration électrophysiologique, panne de micromoteur, (un patient, 0,5\%); panne de neurostimulateur externe (un patient, $0,5 \%$ ).

\section{Discussion}

Cette étude rétrospective des EI non stimulo-induits de la chirurgie montre qu'un EI au moins est survenu pour $28 \%$ des patients, et qu'il s'agissait d'un EI grave dans 6,8\%. Le taux d'EI était plus élevé lors du positionnement des électrodes, que lors de l'implantation du neurostimulateur ou que lors de la réalisation de l'IRM. Les EI graves sont survenus identiquement lors de la mise en place des électrodes ou du neurostimulateur ; néanmoins, le seul décès a été lié à la procédure d'implantation d'électrode.

Nos résultats sont similaires à ceux de la littérature, tant pour le risque général en neurochirurgie (Bruder et al., 2007 ; Manninen et al., 1999) que ceux de la SCP (Hariz et al., 2008 ; Joint et al., 2002 ; Lyons et al., 2004 ; Oh et al., 2002 ; Seijo et al., 2007 ; Umemura et al., 2003). Le taux d'hémorragie intracrânienne dans cette série est faible $(0,5 \%)$ bien que comparable à celui de la littérature qui est inférieur à $2 \%$ (Lyons et al., 2004 ; Umemura et al., 2003) ; cette complication peut être à l'origine de séquelles graves (Lyons et al., 2004 ; Umemura et al., 2003). En général, le saignement est cortical, au point d'entrée de l'électrode, visible sur l'imagerie postopératoire immédiate, mais sans retentissement clinique. Il peut être minimisé en évitant les vaisseaux au point d'entrée visibles sur l'IRM pré opératoire, en travaillant à dure mère fermée, sans déplacement du parenchyme cérébral, ni baisse des pressions intra crâniennes, et en limitant le nombre d'électrodes d'exploration électrophysiologique. Le nombre de complications hémorragiques est insuffisant dans notre série pour pouvoir conclure formellement à l'influence de l'âge ou de la cible visée, ni du nombre de trajectoires d'électrophysiologie.

L'utilisation d'un kit de repositionnement permet de réduire le temps opératoire et améliore le confort du patient. La réduction du temps opératoire permet aussi, associée à une diminution de la durée d'exploration électrophysiologique, de limiter la survenue de confusion per opératoire, et de troubles respiratoires, chez les patients, âgés, fragiles physiologiquement et à jeun de leur traitement. La réduction du temps opératoire permet aussi de minimiser le risque infectieux (Lyons et al., 2004).

Dans notre série, nous n'avons pas eu à déplorer de déplacement ou " shift » cérébral, ni de déplacement post opératoire d'électrodes. La technique de travail à dure mère fermée, et les systèmes d'étanchéité et de fixation l'électrode à la voûte avant son largage, peuvent peut être permettre d'éviter ces complications rapportées par d'autres équipes (Oh et al., 2002 ; Umemura et al., 2003).

Les complications liées à l'implantation du neurostimulateur sont liées au fait qu'il s'agit d'un matériel implantable et qu'il faut tunnéliser les prolongateurs à l'aveugle, en région cervicale, pouvant léser une veine jugulaire externe. Le risque hémorragique sur le site d'implantation du neurostimulateur est limité par une hémostase rigoureuse de la poche sous-cutanée. Le risque infectieux est réduit par l'utilisation d'une antibioprophylaxie per opératoire (Martin, 2000). Enfin, il est recommandé d'implanter le 
stimulateur dans la région sou-claviculaire, et de le fixer à l'aponévrose pour limiter le risque de migration et de tension sur les prolongateurs. Cependant, compte tenu du volume des neurostimulateurs double canal et pour des raisons esthétiques, nous avons été contraints d'implanter le générateur dans la région abdominale. Dans notre série, le site d'implantation n'influençait pas la gêne potentielle occasionnée par le neurostimulateur.

Les problèmes techniques liés au matériel chirurgical, cadre et micromoteur, peuvent être réduits en assurant un meilleur contrôle qualité avant son utilisation, et en respectant les recommandations fournis par le fabricant. Les problèmes liés à l'IRM stéréotaxique sont principalement dus aux troubles de la statique rachidienne qui peuvent rendre difficile l'alignement du cadre avec son boîtier de repérage dans l'antenne tête. Avec l'expérience, cette limitation peut être contournée en compensant les déformations rachidiennes par des appuis appropriés.

Nous n'avons pas dans notre série à déplorer d'incidents suite à la réalisation d'IRM postopératoire. L'Afssaps et la société Medtronic recommande de ne pas dépasser une énergie de $0,1 \mathrm{~W} / \mathrm{kg}$, pour éviter un échauffement autour des électrodes intracérébrales (Henderson et al., 2005 ; Nutt et al., 2001 ; Roark et al., 2008). Nous ne réalisons donc plus que des contrôles tomodensitométriques postopératoires immédiats (Hemm et al., 2009).

Enfin, comme pour toute technique chirurgicale, les risques d'EI diminuent au cours du temps (Fig. 1), ceci par amélioration des pratiques et évolutions techniques.

\section{Conclusion}

La SCP reste un acte chirurgical dont le nombre d'EI non stimulo-induits liés à la chirurgie n'est pas négligeable. Les EI graves sont rares. Un certain nombre de ces complications est sûrement explicable par la fragilité physiologique provoquée par les maladies neurologiques. Les autres complications devraient pouvoir être réduites en raccourcissant les temps opératoires (équipe chirurgicale entraînée, ciblage anatomique primaire et diminution du nombre d'exploration électrophysiologique) et en améliorant les matériels, de l'appareillage de neurostimulation jusqu'aux instruments d'implantation.

Déclaration d'intérêts Les auteurs déclarent ne pas avoir de conflits d'intérêts en relation avec cet article. 


\section{Références}

S. Blond, G. Touzet, P. Krystkowiak, L. Defebvre, A. Deste Neurosurgical treatment of Parkinson's disease: which technique is best? Rev. Neurol. (Paris), 156 (Suppl 2 Pt 2) (2000), pp. 257-269

N.R. Bruder, Patrick, Ravussin Complications postopératoires en chirurgie réglée. La réanimation neurochirurgicale S. Verlag, Paris (2007) pp. 301-316

G. Deuschl, C. Schade-Brittinger, et al. Neurostimulation Section. A randomized trial of deep-brain stimulation for Parkinson's disease N. Engl. J. Med., 355 (9) (2006), pp. 896-908

M.I. Hariz, S. Rehncrona, N.P. Quinn, J.D. Speelman, C. Wensing Multicenter study on deep brain stimulation in Parkinson's disease: an independent assessment of reported adverse events at 4 years Mov. Disord., 23 (3) (2008), pp. 416-421

S. Hemm, J. Coste, J. Gabrillargues, L. Ouchchane, L. Sarry, F. Caire, F. Vassal, C. Nuti, P. Derost, F. Durif, J.J. Lemaire Contact position analysis of deep brain stimulation electrodes on post-operative CT images Acta. Neurochir. (Wien), 151 (7) (2009), pp. 823-829

Henderson, J.M., Tkach, J., Phillips, M., Baker, K., Shellock, F.G., Rezai, A.R., 2005. Permanent neurological deficit related to magnetic resonance imaging in a patient with implanted deep brain stimulation electrodes for Parkinson's disease: case report. Neurosurgery 57 (5), E1063.

Joint, C., Nandi, D., Parkin, S., Gregory, R., Aziz, T., 2002. Hardware-related problems of deep brain stimulation. Mov Disord 17 (Suppl 3), S175-S180.

P. Krack, A. Batir, N. Van Blercom, S. Chabardes, V. Fraix, C. Ardouin, A. Koudsie, P.D. Limousin, A. Benazzouz, J.F. LeBas, A.L. Benabid, P. Pollak Five-year follow-up of bilateral stimulation of the subthalamic nucleus in advanced Parkinson's disease N. Engl. J. Med., 349 (20) (2003), pp. 1925-1934 
J.J. Lemaire, F. Caire, J.M. Bony, J.L. Kemeny, A. Villeger, J. Chazal Contribution of 4.7 Tesla MRI in the analysis of the MRI anatomy of the human subthalamic area Acta. Neurochirurgica, 146 (2004), pp. 906-907

J.J. Lemaire, J. Coste, L. Ouchchane, S. Hemm, P. Derost, M. Ulla, S. Siadoux, J. Gabrillargues, F. Durif, J. Chazal MRI anatomical mapping and direct stereotactic targeting in the subthalamic region: functional and anatomical correspondence in Parkinson's disease Int. J. Cars., 2 (2007), pp. 75-85

J.J. Lemaire, F. Durif, J.Y. Boire, B. Debilly, B. Irthum, J. Chazal Direct stereotactic MRI location in the globus pallidus for chronic stimulation in Parkinson's disease Acta. Neurochir. (Wien), 141 (7) (1999), pp. 759-765

K.E. Lyons, S.B. Wilkinson, J. Overman, R. Pahwa Surgical and hardware complications of subthalamic stimulation: a series of 160 procedures Neurology, 63 (4) (2004), pp. 612-616

P.H. Manninen, S.K. Raman, K. Boyle, H. El-Beheiry Early postoperative complications following neurosurgical procedures Can. J. Anaesth., 46 (1) (1999), pp. 7-14

C. Martin, Working Group of Experts of the Societe Francaise d'Anesthesie et de Reanimation (SFAR) Guidelines for antibiotic prophylaxis in surgery patients: application to Neurosurgery. 1999 update Neurochirurgie, 46 (4) (2000), pp. 402-405

T.J. Moore, M.R. Cohen, C.D. Furberg Serious adverse drug events reported to the Food and Drug Administration, 1998-2005 Arch. Intern. Med., 167 (16) (2007), pp. 1752-1759

J.G. Nutt, V.C. Anderson, J.H. Peacock, J.P. Hammerstad, K.J. Burchiel DBS and diathermy interaction induces severe CNS damage Neurology, 56 (10) (2001), pp. 1384-1386

M.Y. Oh, A. Abosch, S.H. Kim, A.E. Lang, A.M. Lozano Long-term hardware-related complications of deep brain stimulation Neurosurgery, 50 (6) (2002), pp. 1268-1274 
P. Plaha, Y. Ben-Shlomo, N.K. Patel, S.S. Gill Stimulation of the caudal zona incerta is superior to stimulation of the subthalamic nucleus in improving contralateral parkinsonism Brain, 129 (Pt 7) (2006), pp. 1732-1747

Roark, C., Whicher, S., Abosch, A., 2008. Reversible neurological symptoms caused by diathermy in a patient with deep brain stimulators: case report. Neurosurgery 62 (1), E256

M.C. Rodriguez-Oroz, J.A. Obeso, A.E. Lang, et al. Bilateral deep brain stimulation in Parkinson's disease: a multicentre study with 4 years follow-up Brain, 128 (Pt 10) (2005), pp. 2240-2249

F.J. Seijo, M.A. Alvarez-Vega, J.C. Gutierrez, F. Fdez-Glez, B. Lozano Complications in subthalamic nucleus stimulation surgery for treatment of Parkinson's disease. Review of 272 procedures Acta. Neurochir. (Wien), 149 (9) (2007), pp. 867-875

A. Umemura, J.L. Jaggi, H.I. Hurtig, A.D. Siderowf, A. Colcher, M.B. Stern, G.H. Baltuch Deep brain stimulation for movement disorders: morbidity and mortality in 109 patients J. Neurosurg., 98 (4) (2003), pp. $779-784$ 